\title{
Surface plasmon resonances in liquid metal nanoparticles
}

\author{
A. E. Ershov ${ }^{1,2,3}$, V. S. Gerasimov ${ }^{2}$, A. P. Gavrilyuk ${ }^{1,2}$, S. V. Karpov Gra, $^{2,3,4}$ \\ 1 Institute of Computational Nodeling, Federal Research Center KSC SB RAS Lrasnoyarsk, 660036, Russia \\ 2 Institute of Nanotechnology, Spectroscopy and Quantum Chemistry, Siberian Federal Cniversity, Krasnoyarsk, 660041, \\ Russia \\ 3 Siberian State Acrospace University, 660011 , Krasnoyarsk, Russia \\ 4 L.V. Kirensky Institute of Physics, Federal Research Center KSC SB RAS, 660036, Krasnoyarsk, Russia
}

\begin{abstract}
We have shown significant suppression of resonant propertins of metallic nanoparticles at the surface plasmon frequency during the phase transition "solidliquid" in the basic materials of nanoplasmonics ( $\mathrm{Ag}$, Au). Using experimental values of the optical constants of liquid and solid metals we have calculated nanoparticle plasmonic absorption spectra. The effect was demonstrated for single particles, dimers and trimers, as well as for the large multiparticle colloidal aggregates. Experimental verification performed for single Au nanoparticles heated to the melting temperature and above up to full suppression of the surface plasmon resonance. It is emphasized that this eflect may underlie the non-linear optical response of composite matcrials containing plasmonic nanoparticles and their aggregates.
\end{abstract}

\section{Introduction}

Interaction of metal nanoparticles with laser radiation is one of the major research areas in nanoplasmonics. I'lasmonic nanoparticles producing strongly localized and enhanced dectromagnetic ficlds have numorous applications. The study of such processes is of topical interest for nanosensorics; biomedicine and biotechnology: in laser excitation of plasmon-polaritons in waveguiding chains of plasmonic nanoparticles, photochromic reactions induced by laser excitation of resonant domains in disordered colloidal nanoparticle aggregates, various nonlinear optical processes etc. Monographs and reviews [1-5] cover a large number of papers published in recent years.

$\Lambda$ special case of these processes takes place at high temperatures when plasmonic nanoparticles are in a liquid state. For example, the effect of laser radiation on alkali metal vapors, produced in heat pipes and applied for nonlinear-optical frequency conversion $[6]$ can be accompanied by formation of a light scattering aerosol subsystem consisting of liquid nanodrops as well as in lighting bulbs containing metal vapors which may scatter and attenuate the radiation.

There are a number of papers (see e.g. $|7-9|$ ) on the heating of nanoparticles by laser radiation. IIowever, these studies do not take into account the effect of heating on optical properties of namoparticles and wice cersa.

Authors of the paper $[10]$ have shown that the dependence of optical properties of gold nanorods on their temperature, changing under the influence of high-intensity pulsed laser radiation results in formation of non-linear optical response of such systems.

In our earlier papers $[11-13]$ we studied the influence of pulsed laser radiation on plasmonic Ag nanoparticle aggregates and took into account the effect of molting of nanoparticles on their resonant properties.

This factor can be accompanied by an unusual feature: a cyclically repeating rise and fall of the temperature of resonantly excited nanoparticles within a nanosecond laser pulse. The cycles occur due to cease of resonant interaction of radiation with liquid particles having suppressed surface plasmon resonance (SPR) and following recovery of $S L^{\prime} R$ during cooling and subsequent reheating.

Suppression of SPR can induce optical nonlinearity of nanocolloids and composite matcrials containing plasmonic nanoparticles and their aggregates in highintensity laser fields.

The goal of our work is to study the interaction between optical radiation and plasmonic nanoparticles under their extreme heating and melting and to andyze the resulting changes in their optical properties using theoretical and experimental methods.

\section{Model}

Changes in the optical properties of the particle material due to melting are an important factor to be considered when studying the interaction between laser radiation and nanoparticles. Iet us look at it in more detail. The 
intensity of phonon oscillations grows with the particle temperature that increases the frequency of scattering of electrons at phonons. This results in an increased electron relaxation constant (above the Debye temperature, the electron relaxation grows in proportion to the first power of the temperature) [14]. Gradual destruction of the periodic structure in a melting crystal leads to scattering of the conduction electrons at the lattice defects up to complete amorphization. Melting is accompanied by an abrupt increase in the relaxation constants. Apart from phonons, contribution to the electron relaxation of metal is determined by electron scattering at point defects, dislocations, grain boundaries [14].

\subsection{The heating of nanoparticles and the kinetics of their thermodynamic characteristics}

As mentioned above, heating of nanoparticles when exposed to laser radiation is an important factor causing changes in optical characteristics of the particles. The absorbed power of laser radiation $\left(W_{i}\right)$ in the dipole approximation is described by the equation [15]:

$$
W_{i}=\frac{\omega\left|\mathbf{d}_{i}\right|^{2}}{2 \epsilon_{0}} \operatorname{Im}\left(\frac{1}{\alpha_{i}^{*}}\right),
$$

where $\omega$ is the frequency of laser radiation, $\mathbf{d}_{i}$ is the vector of the dipole moment of a particle and will be determined below in eq. (19), $\alpha_{i}$ is the dipole polarizability of the $i$-th particle (see eq. 15 ), symbol $*$ denotes complex conjugation, $\epsilon_{0}$ is the electric constant.

The absorption of laser radiation leads primarily to heating of conduction electrons in nanoparticles and their ion subsystem (crystal lattice). The temperature change in the electron subsystem due to absorption of the electromagnetic wave energy and heat exchange with the ion subsystem is described by the equation $[16,17]$

$$
\left(C_{\mathrm{e}}\right)_{i} \frac{d\left(T_{\mathrm{e}}\right)_{i}}{d t}=-g\left[\left(T_{\mathrm{e}}\right)_{i}-\left(T_{\mathrm{i}}\right)_{i}\right]+\frac{W_{i}}{V_{i}} .
$$

Here $\left(C_{e}\right)_{i}$ is the volumetric heat capacity of electron subsystems [11]: $\left(C_{e}\right)_{i}=68\left(T_{i}\right)_{i} \mathrm{~J} \cdot \mathrm{m}^{-3} \mathrm{~K}^{-1},\left(T_{\mathrm{i}}\right)_{e}$ and $\left(T_{\mathrm{i}}\right)_{i}$ are the temperatures of the electron and ion subsystem, $V_{i}$ is the particle volume, $g=4 \cdot 10^{16} \mathrm{~J} \cdot \mathrm{m}^{-3} \mathrm{~K}^{-1} \mathrm{~s}^{-1}$ is the temperature independent rate of energy exchange between the electron and ion subsystems above the Debye temperature [18-20].

The temperature of the ion subsystem of a particle $\left(T_{\mathrm{i}}\right)_{i}$ changes under heat exchange between the electron subsystem and the environment. To take into account the "solid-liquid" phase transition in particles we use, for convenience, the equation for the thermal energy of the lattice $\left(Q_{\mathrm{i}}\right)_{i}$ rather than the equation for $T_{\mathrm{i}}$ :

$$
\frac{d\left(Q_{\mathrm{i}}\right)_{i}}{d t}=g V_{i}\left[\left(T_{\mathrm{e}}\right)_{i}-\left(T_{\mathrm{i}}\right)_{i}\right]+\left(q_{\mathrm{l}}\right)_{i} V_{i}
$$

where $\left(q_{1}\right)_{i}$ is the heat flux per unit volume describing thermal losses [16]:

$$
\left(q_{1}\right)_{i}=-\frac{3}{2 R_{i}}\left(\chi_{\mathrm{m}} c_{\mathrm{m} 0} \rho_{\mathrm{m}}\right)^{1 / 2} \cdot\left[\left(T_{\mathrm{i}}\right)_{i}-T_{0}\right] \cdot t^{-1 / 2},
$$

$\chi_{\mathrm{m}}$ is the thermal conductivity of the interparticle medium, $c_{\mathrm{m} 0}$ is its heat capacity, and $\rho_{\mathrm{m}}$ is the density, $t$ is the time since the beginning of a pulse, $T_{0}$ is the temperature of the interparticle medium.

The temperature of the ion subsystem taking into account the melting process, is expressed via $\left(Q_{\mathrm{i}}\right)_{i}$ :

$$
\begin{gathered}
\left(T_{\mathrm{i}}\right)_{i}=\frac{\left(Q_{\mathrm{i}}\right)_{i}}{C_{i} V_{i}} H\left(\left(Q_{1}\right)_{i}-\left(Q_{i}\right)_{i}\right)+ \\
\frac{\left(Q_{\mathrm{i}}\right)_{i}-\left(Q_{2}\right)_{i}}{C_{i} V_{i}} H\left(\left(Q_{\mathrm{i}}\right)_{i}-\left(Q_{2}\right)_{i}\right)+ \\
T_{\mathrm{m}}\left(R_{i}\right) H\left(\left(Q_{\mathrm{i}}\right)_{i}-\left(Q_{1}\right)_{i}\right),
\end{gathered}
$$

where $\left(Q_{1}\right)_{i}$ and $\left(Q_{2}\right)_{i}$ are the particle thermal energies, at the beginning and the end of melting, respectively, $C_{i}$ is the volumetric heat capacity of the ion subsystem of particles, $T_{\mathrm{m}}\left(R_{i}\right)$ is the melting point, which depends on the particle size [21], $R_{i}$ is the particle radius, $H(x)$ is the Heaviside function.

\subsection{Manifestation of relaxation processes in plasmonic nanoparticles during their heating and melting}

Relaxation processes in nanoparticles determine the spectral width of SPR. In this section we employ different approaches to estimate the equilibrium magnitude of the relaxation constant of conduction electrons for the nanoparticle material $(\Gamma)$ and to analyze the reasons for its variations in different publications. In addition, we determine the values of the Drude approximation parameters to describe adequately the experimental spectral dependence of dielectric constants.

In the case of a single particle, as follows from eq. (1), the absorbed radiation power $(W)$ depends on the dielectric constant $\varepsilon=\varepsilon^{\prime}+\mathrm{i} \varepsilon^{\prime \prime}$ of the nanoparticle material and the surrounding medium. Extracting and omitting from eq. (1) all terms except the frequency-dependent ones taking into account eq. (15) and (16) for homogeneous particles (solid or liquid) we define this part of the function $F(\omega)$ as

$$
W \propto F(\omega)=\varepsilon_{\mathrm{m}}^{3 / 2} \frac{\omega \varepsilon^{\prime \prime}}{\left(\varepsilon^{\prime}+2 \varepsilon_{\mathrm{m}}\right)^{2}+\varepsilon^{\prime \prime 2}},
$$

where $\varepsilon_{\mathrm{m}}$ is permittivity of the medium. A particle absorbing radiation is heated up to a complete melting. If the temperature and the state of aggregation change, the dielectric constant of the material changes as well [22-24]. This affects the radiation absorption [25, 26], which should be taken into account when studying the interaction with laser radiation. One of the main reasons for the change of a dielectric constant is an increase in 


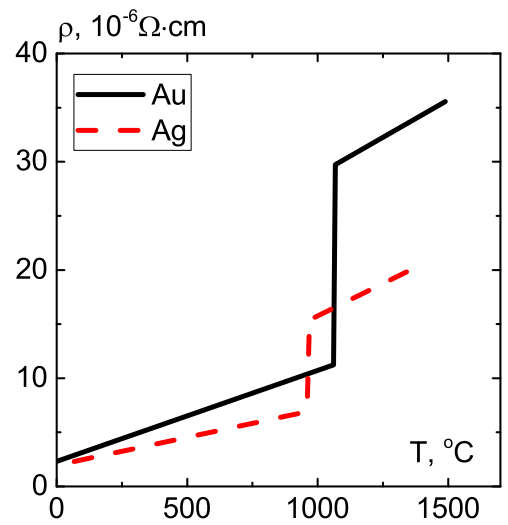

Fig. 1 Specific resistance of metals during transition from solid to liquid state [27]

the relaxation constant of the electron subsystem (taking into account the size a a particle and the finite size effect):

$$
\Gamma_{\mathrm{e}-\mathrm{ph}, \mathrm{d}}(T, R)=\Gamma_{\mathrm{e}-\mathrm{ph}, \mathrm{d}}(T)+A \frac{v_{f}}{R},
$$

where $\Gamma_{\mathrm{e}-\mathrm{ph}, \mathrm{d}}(T)$ is the relaxation constant corresponding to the frequency of scattering of conduction electrons at phonons and lattice defects, $v_{f}$ is the Fermi velocity, $R$ is the particle radius, and $A$ is the constant assumed to be equal to 1 in most of the optical calculations. Note that the term temperature hereinafter refers to the crystal lattice temperature.

If we have the dependence $\Gamma_{\mathrm{e}-\mathrm{ph}, \mathrm{d}}(T)$ we can express the dielectric constant in the Drude approximation of the particle material taking into consideration the particle radius:

$$
\begin{aligned}
\varepsilon(\omega, T, R) & =\varepsilon^{\prime}(\omega, T, R)+\mathrm{i} \varepsilon^{\prime \prime}(\omega, T, R) \\
& =\varepsilon_{0}(\omega)-\frac{\omega_{\mathrm{p}}^{2}}{\omega^{2}+\mathrm{i} \omega \Gamma_{\mathrm{e}-\mathrm{ph}, \mathrm{d}}(T, R)} .
\end{aligned}
$$

Here $\varepsilon_{0}(\omega)$ is the factor that takes into account the contribution of interband transitions to the dielectric constant, $\omega_{\mathrm{p}}$ is the plasma frequency of the metal. Using the available experimental and theoretical data for a bulk $\left(\Gamma_{\mathrm{e}-\mathrm{ph}, \mathrm{d}}(T, R) \rightarrow \Gamma_{\mathrm{e}-\mathrm{ph}, \mathrm{d}}(T)\right.$ according to eq. $\left.(7)\right)$ we find $\Gamma_{\mathrm{e}-\mathrm{ph}, \mathrm{d}}(T)$ for $\mathrm{Ag}$ and $\mathrm{Au}$.

Consider the case of silver. The dielectric constants $\varepsilon^{\prime}$ and $\varepsilon^{\prime \prime}$ at room temperature $T_{\mathrm{r}}=273 \div 300 \mathrm{~K}$ were obtained for Ag films in a number of papers (e. g. [22,28,29], and those for liquid metal at the melting point can be found in [23]. Unfortunately, the temperature dependences of the dielectric constants obtained in [23] are outside the spectral range of SPR for silver and therefore cannot be used for Ag nanoparticles.

In the Drude approximation, based on the results from $[22,28,29]$ we have $\Gamma_{\mathrm{e}-\mathrm{ph}, \mathrm{d}}\left(T_{\mathrm{r}}\right) \simeq(1 \div 3) \cdot 10^{14} \mathrm{~s}^{-1}$ (ignoring relaxation at the lattice defects at $T_{\mathrm{r}}$ ). At the same time, the specific resistance for silver gives the value $\Gamma_{\mathrm{e}-\mathrm{ph}, \mathrm{d}}\left(T_{\mathrm{r}}\right) \simeq 2.6 \cdot 10^{13} \mathrm{~s}^{-1}$ (see $\left.[30,31]\right)$ Nearly the

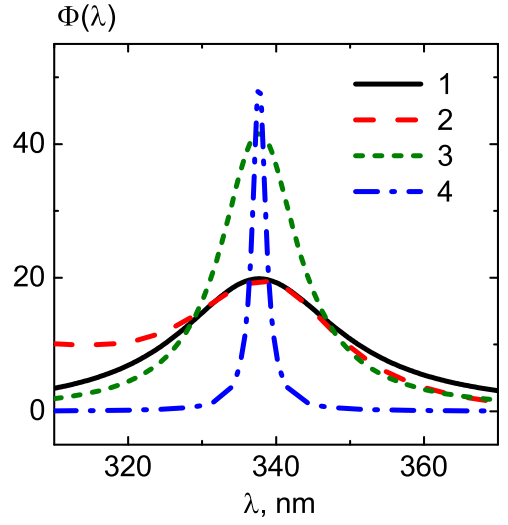

Fig. 2 Dependence of the relative absorption of $\mathrm{Ag}$ particle $\Phi(\lambda)$ vs wavelength for $1-T=1233 \mathrm{~K}$ (liquid), $2-$ according to the experimental results [23], $3-T=1233 \mathrm{~K}$ (solid), $4-T=273 \mathrm{~K}$ (this curve is shown as $\Phi(\lambda) / 5$ ).

same values were obtained in $[28,31,32]$ in the Drude approximation for the infrared frequency range $\Gamma_{\mathrm{e}-\mathrm{ph}, \mathrm{d}}\left(T_{\mathrm{r}}\right)$ $\simeq(2.8 \div 4) \cdot 10^{13} \mathrm{~s}^{-1}$. Such a significant difference, in our opinion, may be attributed to the additional contribution to the relaxation constant of solid metal bulk such factors as the surface roughness and polycrystalline structure $[33,34]$. To take into consideration these factors (including the electron-phonon scattering), the papers above introduce the notion of an effective relaxation constant $\Gamma_{\text {eff }}$, which can significantly exceed $\Gamma_{\mathrm{e}-\mathrm{ph}, \mathrm{d}}\left(T_{\mathrm{r}}\right)$. For example, it is shown in Ref. [33] that the surface roughness increases the relaxation constant up to $\Gamma_{\text {eff }} \simeq$ $1.6 \cdot 10^{14} \mathrm{~s}^{-1}$, which well agrees with the data experimentally obtained in [28]. However in the case of smaller particles $(R \leq 30 \mathrm{~nm}$ ) and liquid films most of the factors listed above can be ignored and the scattering of electrons at phonons and lattice defects is dominant factor in the relaxation constant (in addition to the finite size effect). Therefore, we will use the results from [23], with the corresponding selected parameters of the Drude approximation for liquid silver (denoted $l$ ) at melting temperature $T_{\mathrm{m}}: \omega_{\mathrm{p}}=1.33 \cdot 10^{16} \mathrm{~s}^{-1}, \Gamma_{\mathrm{e}-\mathrm{ph}, \mathrm{d}}^{(l)}\left(T_{\mathrm{r}}\right)=$ $4.4 \cdot 10^{14} \mathrm{~s}^{-1}, \varepsilon_{0}=3.65$. Here, due to the interband absorption wing being separated from the SPR band in the absorption spectra the term $\varepsilon_{0}(\omega)$ can be taken constant. Considering that $\Gamma_{\mathrm{e}-\mathrm{ph}, \mathrm{d}}(R, T)$ is proportional to the specific electrical resistivity $\rho$ and abruptly increases by 2.1 times for silver during melting [27] (Fig. 1) we can assume that the relaxation constant for a solid sample (denoted as " $s$ ") will be equal to $\Gamma_{\mathrm{e}-\mathrm{ph}, \mathrm{d}}^{(s)}\left(T_{\mathrm{m}}\right)=$ $4.4 \cdot 10^{14} / 2.1 \approx 2.1 \cdot 10^{14} \mathrm{~s}^{-1}$.

At temperatures below $T_{\mathrm{m}} \Gamma_{\mathrm{e}-p h, d}(T) \sim T$ (see [35]). To find this temperature dependence we use the following equation $[24,35]$ :

$$
\Gamma_{\mathrm{e}-\mathrm{ph}, \mathrm{d}}(T)=K_{0} T^{5} \int_{0}^{\theta / T} \frac{z^{4} d z}{e^{z}-1},
$$




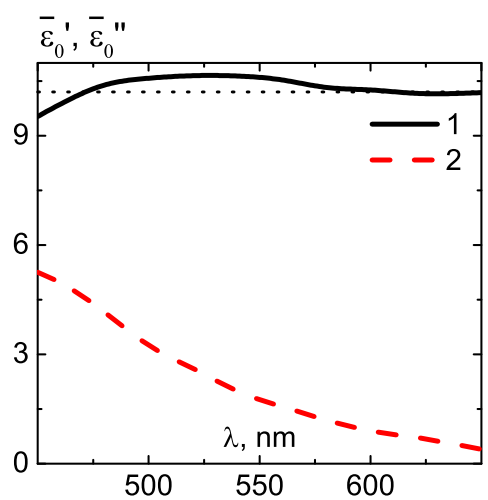

Fig. 3 The spectral dependence of the interband absorption factor in dielectric constant averaged over temperatures: 1 $\bar{\varepsilon}_{0}^{\prime}, 2-\bar{\varepsilon}_{0}^{\prime \prime}$.

where $\theta=225 \mathrm{~K}$ is the Debye temperature for silver. The coefficient $K_{0}$ can be found using eq. (4) for already determined $\Gamma_{\mathrm{e}-\mathrm{ph}, \mathrm{d}}^{(s)}\left(T_{\mathrm{m}}\right)$. Eq. (1) nearly perfectly describes the linear dependence in the temperature range $(273 \div 1233 \mathrm{~K})$ :

$$
\begin{aligned}
\Gamma_{\mathrm{e}-\mathrm{ph}, \mathrm{d}}(T) & =A_{A g} \cdot T+B_{A g}, \\
A_{A g} & =1.81 \cdot 10^{11} \mathrm{~K}^{-1} \cdot \mathrm{s}^{-1}, \\
B_{A g} & =1.45 \cdot 10^{13} \mathrm{~s}^{-1} .
\end{aligned}
$$

In particular, it implies that at room temperature $(273 \div 300 \mathrm{~K})$, the relaxation constants are equal to $\Gamma_{\mathrm{e}-\mathrm{ph}, \mathrm{d}}(T=273 \mathrm{~K})=3.5 \cdot 10^{13} \mathrm{~s}^{-1} \quad$ and $\Gamma_{\mathrm{e}-\mathrm{ph}, \mathrm{d}}(T=$ $300 \mathrm{~K})=4.0 \cdot 10^{13} \mathrm{~s}^{-1}$, which is in agreement with the estimates from other publications $[28,31,32]$. Fig. 2 shows the relative absorption (eq. (1)) vs wavelength, for different temperatures and different states of aggregation of $\mathrm{Ag}$ nanoparticles $(\Phi(\lambda)$ corresponds to $F(\omega)$ taking into account substitution of $\omega$ by $\lambda: F(\omega)=F(2 \pi c / \lambda)$ ). Note, that a further temperature growth $(T>1233 \mathrm{~K})$ is accompanied by spectral changes. As we can see from this figure, the absorption of radiation by a nanoparticle during its heating and subsequent melting significantly drops in comparison with room temperature.

Consider the case of gold. Here we deal with a somewhat different situation. Firstly, the SPR band is not separated from the interband absorption band, these two bands partially overlap with each other. Secondly, there are experimental results available in [22] with the temperature dependence of optical constants for the wavelength range (460-650 $\mathrm{nm}$ ) covering the SPR band.

Therefore, using eq. (8) for $\varepsilon_{0}(\omega)$, we assume that the interband absorption depends on the radiation frequency and is described by the expression (for a bulk)

$$
\varepsilon_{0}(\omega)=\varepsilon(\omega, T)+\frac{\omega_{\mathrm{p}}^{2}}{\omega^{2}+\mathrm{i} \omega \Gamma_{\mathrm{e}-\mathrm{ph}, \mathrm{d}}(T)} .
$$

Eq. (11) includes the Drude addition containing the function $\Gamma_{\mathrm{e}-\mathrm{ph}, \mathrm{d}}(T)$ that we need to find. To that end

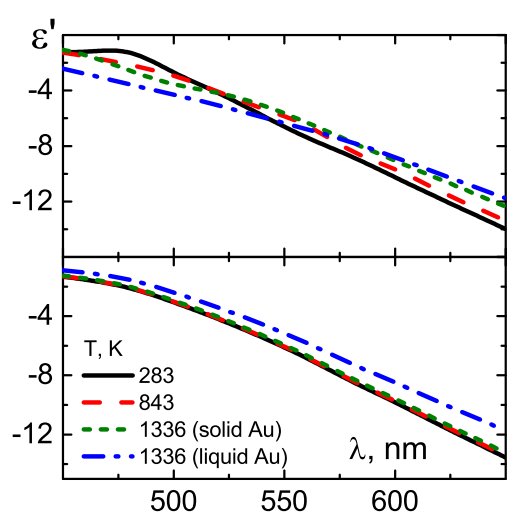

(a)

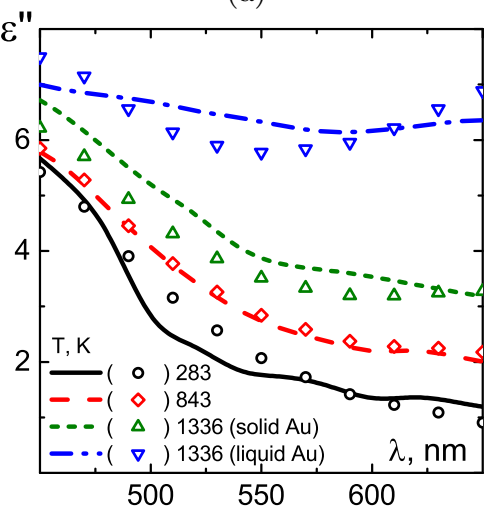

(b)

Fig. 4 Spectral dependences of dielectric constants real (a) and imaginary parts (b): experimental (top in (a) and lines in (b)) [22] and calculated ones (bottom for (a) and scatter plot in (b)) at different temperatures (see the legend).

we will use eq. (9), which requires data on the value of $\Gamma_{\mathrm{e}-\mathrm{ph}, \mathrm{d}}(T)$ at any temperature and we will start from $T=283 \mathrm{~K}[22]$. According to estimates from [28,34] the relaxation constant at room temperature equals $\Gamma_{\mathrm{e}-\mathrm{ph}, \mathrm{d}}$ $\approx 9 \cdot 10^{13} \mathrm{~s}^{-1}$. At the same time the specific resistance for gold [31] provides us with the value $\Gamma_{\mathrm{e}-\mathrm{ph}, \mathrm{d}}=3.65$. $10^{13} \mathrm{~s}^{-1}$. Therefore, based on the same arguments as in the case of silver regarding the influence of various factors on the relaxation constant measured in films (including interband absorption), we choose $\Gamma_{\mathrm{e}-\mathrm{ph}, \mathrm{d}}(T=$ $283 \mathrm{~K})=6 \cdot 10^{13} \mathrm{~s}^{-1}$. Note, that in this case, we again assume that the scattering of electrons at phonons and lattice defects is the dominant relaxation mechanism in nanoparticles. Substituting the selected value in the formula (9), we obtain the dependence which is well described by a linear function below the melting point:

$$
\begin{aligned}
\Gamma_{\mathrm{e}-p h, d}(T) & =A_{A u} \cdot T+B_{A u}, \\
A_{A u} & =2.68 \cdot 10^{11} \mathrm{~K}^{-1} \cdot \mathrm{s}^{-1}, \\
B_{A u} & =1.6 \cdot 10^{13} \mathrm{~s}^{-1} .
\end{aligned}
$$




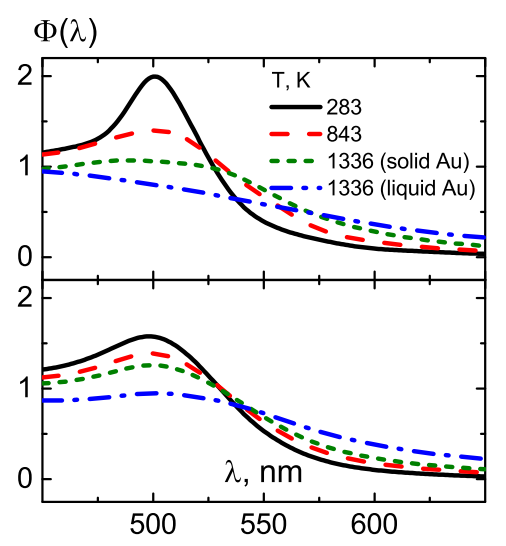

$\Phi(\lambda)$

(a)

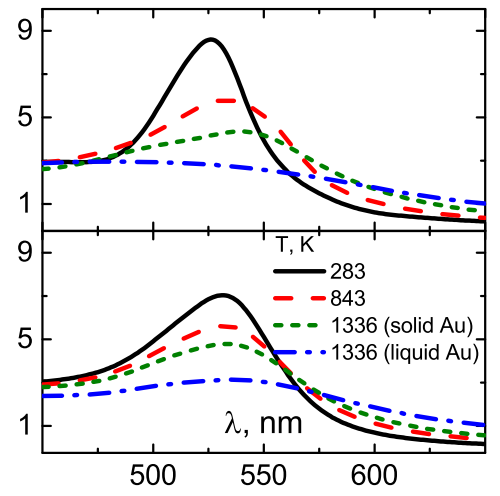

(b)

Fig. 5 The relative absorption spectra of $\mathrm{Au}$ nanoparticles at different temperatures (see legend): top - calculated using the experimental data on dielectric constants [22]; bottom obtained by approximating eq. (8) for a bulk: (a) $-\varepsilon_{\mathrm{m}}=1$, (b) $-\varepsilon_{\mathrm{m}}=2.2$.

Upon melting, the specific resistance of gold exhibits a 2.4 times jump (Fig. 1) resulting in the change of the relaxation constant

$$
\Gamma_{\mathrm{e}-\mathrm{ph}, \mathrm{d}}^{(l)}\left(T_{\mathrm{m}}\right)=2.4 \cdot \Gamma_{\mathrm{e}-\mathrm{ph}, \mathrm{d}}^{(s)}\left(T_{\mathrm{m}}\right) .
$$

Assuming that $\varepsilon_{0}(\omega)$ is independent of temperature, we choose the value $\bar{\varepsilon}_{0}(\omega)=\left\langle\varepsilon_{0}(\omega)\right\rangle_{T}$ averaged over temperatures. As follows from eq. (11), the value of $\varepsilon_{0}$ depends on $\omega_{p}$ which is unknown. To find it we varied the value $\omega_{\mathrm{p}}$ so that $\bar{\varepsilon}_{0}$ was as close as possible to $\varepsilon_{0}(\omega)$. In this way we can determine $\omega_{\mathrm{p}}$ and $\varepsilon_{0}(\omega)$. The obtained plasma frequency is equal to $\omega_{\mathrm{p}}=1.44 \cdot 10^{16} \mathrm{~s}^{-1}$. Fig. 3 shows the spectral dependences of the real $\left(\bar{\varepsilon}_{0}^{\prime}\right)$ and the imaginary $\left(\bar{\varepsilon}_{0}^{\prime \prime}\right)$ parts of the dielectric constant taking into account the contribution from the interband absorption. Note that $\bar{\varepsilon}_{0}^{\prime}$ is nearly frequency independent and slightly varies with respect to its mean value 10.2 . The authors of $[36,37]$ proposed the value 9.5. The dependence of the imaginary part $\bar{\varepsilon}_{0}^{\prime \prime}$ in Fig. 3 is monotonically decreasing. Fig. 4 shows experimental and calculated dependences $\varepsilon^{\prime}(\omega), \quad \varepsilon^{\prime \prime}(\omega)$ at different temperatures.
Having found $\varepsilon(\omega, T)$, we can now find $\Phi(\lambda)$ for gold. Fig. 5 shows $\Phi(\lambda)$ obtained with calculated (top) and experimental (bottom) [22] values of $\varepsilon(\omega, T)$ under the same conditions as in Fig. 4.

We can see that Fig. 5 demonstrates satisfactory qualitative agreement between the theoretical and experimental absorption. Despite a rather low value of $\Gamma_{\mathrm{e}-\mathrm{ph}, \mathrm{d}}(T)$ $=6 \cdot 10^{13} \mathrm{~s}^{-1}$ at room temperature $T=283 \mathrm{~K}$, the width of the SPR peak is fairly large, which is an indication of a significant influence of the interband absorption.

\subsection{Variation of resonant properties of plasmonic nanoparticles and their aggregates during phase transition}

Now we have information on the material characteristics and their behavior in different conditions and we can consider interaction of a plane electromagnetic wave

$$
\mathbf{E}(\mathbf{r})=\mathbf{E}_{0} \exp (\mathrm{ik} \cdot \mathbf{r})
$$

with a single spherical nanoparticle or an aggregate of spherical nanoparticles interacting with each other and with the optical radiation via fields produced by lightinduced dipole moments of other particles. The particle sizes are assumed to be much smaller than the radiation wavelength.

In this model, we consider the case when the core of a metal nanoparticle of radius $R_{i}-h_{i}$ is solid and covered by a liquid metal nanoshell having the thickness $h_{i}$ [38]. This model includes the special cases of a fully solid $\left(h_{i}=0\right)$ and fully liquid particle $\left(h_{i}=R_{i}\right)$. Taking into account the radiation reaction correction the polarizability of a particle $\left(\alpha_{i}\right)$ is described by the equation [39]

$$
\alpha_{i}=\left(\left[\alpha_{i}^{(0)}\right]^{-1}-\frac{2}{3} i|\mathbf{k}|^{3}\right)^{-1},
$$

$$
\begin{aligned}
& \alpha_{i}^{(0)}=4 \pi R_{i}^{3} \times \\
& \frac{\left(\varepsilon_{i}^{(l)}-\varepsilon_{\mathrm{m}}\right)\left(\varepsilon_{i}^{(s)}+2 \varepsilon_{i}^{(l)}\right)+f_{i}\left(\varepsilon_{i}^{(s)}-\varepsilon_{i}^{(l)}\right)\left(\varepsilon_{\mathrm{m}}+2 \varepsilon_{i}^{(l)}\right)}{\left(\varepsilon_{i}^{(l)}+2 \varepsilon_{\mathrm{m}}\right)\left(\varepsilon_{i}^{(s)}+2 \varepsilon_{i}^{(l)}\right)+2 f_{i}\left(\varepsilon_{i}^{(s)}-\varepsilon_{i}^{(l)}\right)\left(\varepsilon_{i}^{(l)}-\varepsilon_{\mathrm{m}}\right)},
\end{aligned}
$$

where $\mathbf{k}$ is the wave vector of laser radiation, $\varepsilon_{i}^{(s)}$ and $\varepsilon_{i}^{(l)}$ are the dielectric constants of the solid and liquid fractions of a particle, $\varepsilon_{\mathrm{m}}$ is a permittivity of surrounding medium, $f_{i}$ is the mass fraction of solid material in the particle (see eq. (21) in [40]). The dielectric constant of metal particles depends on the size of a particle and varies as in eq. (8).

For the extinction (absorption) efficiency of a particle ensemble surrounded by other particles we have the formula [41] (for a single particle, averaging over ensemble 
is omitted):

$$
Q_{\mathrm{e}}=\frac{\sigma_{\mathrm{e}}}{\sum_{i=1}^{N} \pi R_{i}^{2}} .
$$

Here the extinction cross section is given by the equation

$$
\sigma_{\mathrm{e}}=4 \pi|\mathbf{k}| \operatorname{Im} \sum_{i=1}^{N} \frac{\left(\mathbf{d}_{i} \cdot \mathbf{E}^{*}\left(\mathbf{r}_{i}\right)\right)}{\left|\mathbf{E}_{0}\right|^{2}} .
$$

For the $i$-th particle in an aggregate, the light induced dipole moment $\mathbf{d}_{i}$ is modified due to the contribution from other particles to the local field:

$$
\begin{aligned}
& \mathrm{d}_{i \alpha}=\epsilon_{0} \alpha_{i}\left[\left(E_{0}\right)_{\alpha} \exp \left(\mathrm{ikr}_{i}\right)+\sum_{j \neq i}^{N} \sum_{\beta=1}^{3} G_{\alpha \beta}\left(\mathbf{r}^{\prime}{ }_{i j}\right) \mathrm{d}_{j \beta}\right], \\
& \alpha, \beta=x, y, z
\end{aligned}
$$

$\mathbf{d}_{i}$ is the dipole moment of the $i$-th particle in an aggregate interacting with an external optical field and the fields produced by other $(j$-th) particles and obeys the coupled-dipole equations, $\mathbf{r}_{i j}^{\prime}$ is the vector connecting the centers of the particles, adjusted for the renormalization coefficient $[13,41] ; G_{\alpha \beta}$ is the interparticle interaction tensor [41].

\section{Results and discussion}

In this Section we consider how plasmonic absorption spectra of nanoparticles change with variation of the dielectric constant of the particle material and when the melting temperature is reached. The spectral range is limited by the experimental data on the optical constants of liquid metals.

Note that for silver, the Q-factor of a surface plasmon resonance for spherical nanoparticles can be estimated from the ratio $Q_{i}(\omega) \sim\left|\varepsilon^{\prime}\right| / \varepsilon^{\prime \prime}[3]$, which determines the efficiency of interaction of single and bound particles with optical radiation and with each other in an optical field near the resonant frequency.

Such variations of the relaxation constant lead to a change of the optical properties of particles.

Fig. 6 shows the response of the plasmonic absorption of Ag nanoparticle to the change of a thickness of the liquid shell. The dielectric constant for silver is calculated by means of eq. (8) taking into account changes of the relaxation constant.

Fig. 7 shows the temperature kinetics of a $\mathrm{Ag}$ nanoparticle exposed to picosecond laser radiation. One can see that the thermal energy transferred from laser radiation to the particle is quickly stored in the electron subsystem of the metal and gradually transmitted to the ion subsystem even after the pulse is over. Fig. 7 clearly demonstrates the process of melting and crystallization of the particle (two horizontal fragments of the curve).

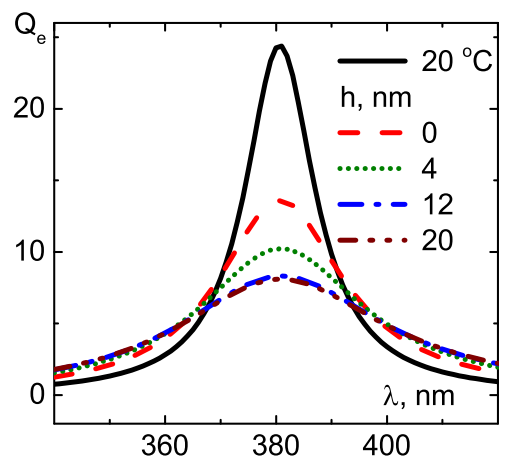

Fig. 6 Surface plasmon absorption spectra of Ag nanoparticle with $R=20 \mathrm{~nm}$ at room temperature and different thicknesses of the liquid metal shell (here and below in Figs 7-11 dielectric constant of the environment $\left.\varepsilon_{\mathrm{m}}=1.78\right)$.)

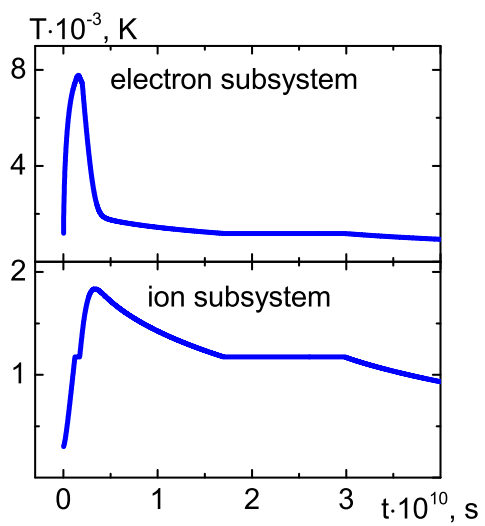

Fig. 7 The kinetics of ion and electron subsystem of $\mathrm{Ag}$ particle with radius $20 \mathrm{~nm}$ when exposed to pulsed laser radiation with duration $\tau=20 \mathrm{ps}$, and the intensity $I=$ $1.663 \cdot 10^{8} \mathrm{~W} \cdot \mathrm{cm}^{-2}$.

It shows that the particle turns into a solid state within 300 ps.

Fig. 8 illustrates the effect of melting of a Au nanoparticle on its SPR (changes of the relaxation constants for $\mathrm{Au}$ have been taken into account).

A comparison of variations of the plasmonic absorption spectra of $\mathrm{Au}$ and $\mathrm{Ag}$ nanoparticles in solid and liquid states in Fig. 6 and Fig. 8 reveals the same tendency, but compared to silver SPR of liquid gold is suppressed stronger.

Figs. 9-11 illustrate variations in the plasmonic absorption spectra of simple $\mathrm{Ag}$ nanoparticle aggregates (dimers, trimers and multiparticle aggregates) when the particles melt under applied pulsed laser radiation. Fig. 9 shows the absorption spectra of a polydisperse Ag dimer calculated by the coupled dipole method. The smaller nanoparticle in a polydisperse dimer melts first, so the high frequency maximum is sufficiently suppressed. Note that despite the larger particle staying solid, its temperature significantly increases, which affects its optical prop- 


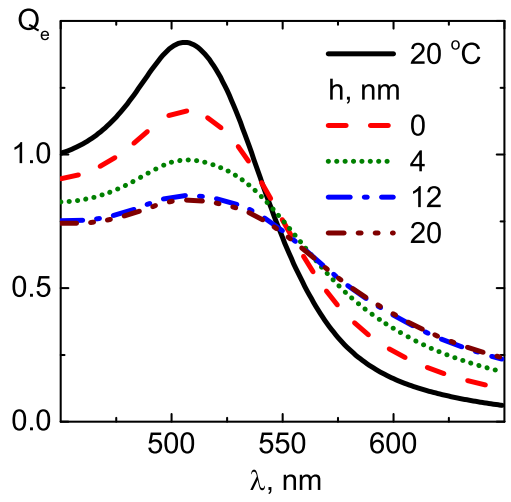

Fig. 8 Plasmonic absorption spectra of Au particle $(R=$ $20 \mathrm{~nm}$ ) with the spectral maximum at $507 \mathrm{~nm}$ at room temperature and during melting up to $(T=1233 \mathrm{~K})$ for different thicknesses of the liquid metal shell.

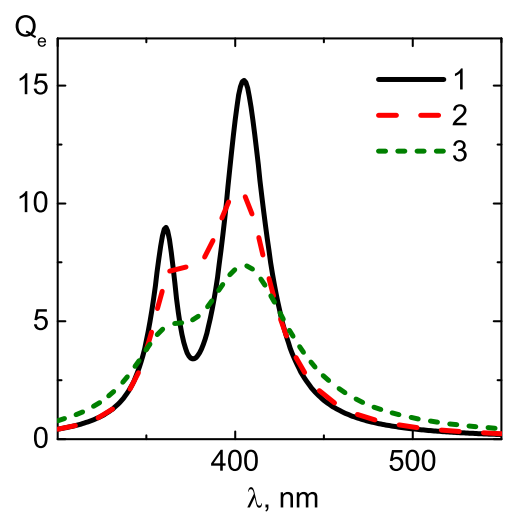

Fig. 9 Absorption spectra of a dimer consisting of Ag nanoparticles with the radiuses 10 and $30 \mathrm{~nm}$ (in Fig. 9 and Fig. 10 the interparticle gap is $4 \mathrm{~nm}$ ). The vector connecting the particle centers is collinear to the radiation polarization. 1 - two particles in a solid state, 2 - the smaller particle in a liquid state, 3 - both particles in a liquid state.

erties. If heated further a larger particle will also melt, causing changes in the absorption spectrum (Fig. 9 (3)).

Fig. 10 shows similar spectra of a monodisperse $\mathrm{Ag}$ trimer. In this trimer, the central particle melts first. As we can see in Fig. 10a (2), this leads to a significant suppression of the resonant property of the trimer. A further heating of the trimer and melting of the side particles (Fig. 10a (3)) leads only to an insignificant suppression of the resonant properties. This is due to the interaction of side particles with the central particle being much stronger than the interaction between the side particles. Therefore, changes in the dielectric properties of the side particles material do not greatly affect the resonant characteristics of the trimer.

The spectral changes of the polydisperse trimer differ from those of the dimer (Fig. 10). In a highly polydisperse trimer the side (smaller) particles melt first. How-

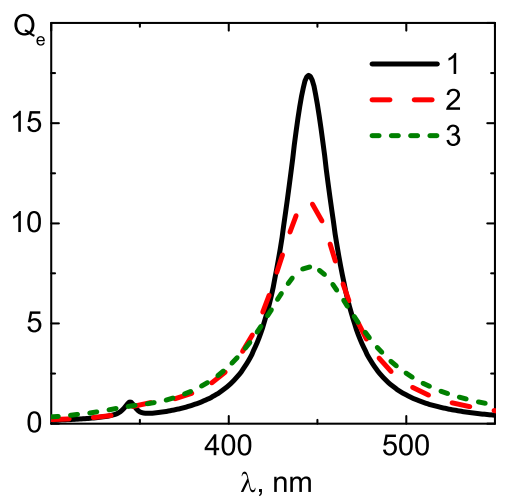

(a)

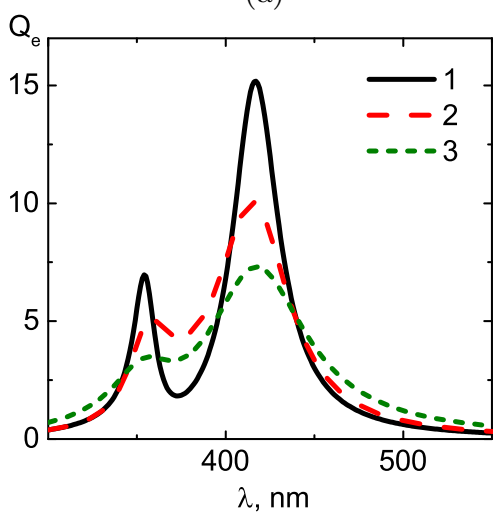

(b)

Fig. 10 (a) - extinction spectra of a trimer consisting of identical Ag nanoparticles with the radius $20 \mathrm{~nm} .1$ - all particles in a solid state, 2 - the central particle in a liquid state, 3 - all particles in a liquid state; (b) - extinction spectra of a trimer, consisting of two particles with the radius $10 \mathrm{~nm}$ (lateral particles) and the central particle with the radius $30 \mathrm{~nm}$; 1 - all particles in a solid state, 2 - side particles is in the liquid state; 3 - all particles in a liquid state. The radiation polarization vector is collinear to the trimer axis.

ever, their melting has little effect on the spectrum of the trimer (Fig. 10b (2)). There is only a small shift of the spectral maximum to shorter wavelengths. Maximal changes in the spectrum occur when the central (larger) particle melts (Figure $10(3)$ ), since it is the central particle makes the largest contribution to the absorption of radiation by the trimer and determines its resonant properties.

In this section we have employed the optodynamic model described in [13] to study the effect of pulsed laser radiation on multiparticle $\mathrm{Ag}$ aggregates with random disordered structure similar to natural ones. This effect is of the following nature: depending on the laser radiation wavelength only a small fraction of particles in an aggregate will undergo melting. These particles belong to resonant domains and their resonant frequency is close to the laser radiation frequency. Under these conditions, understanding the processes responsible for the 


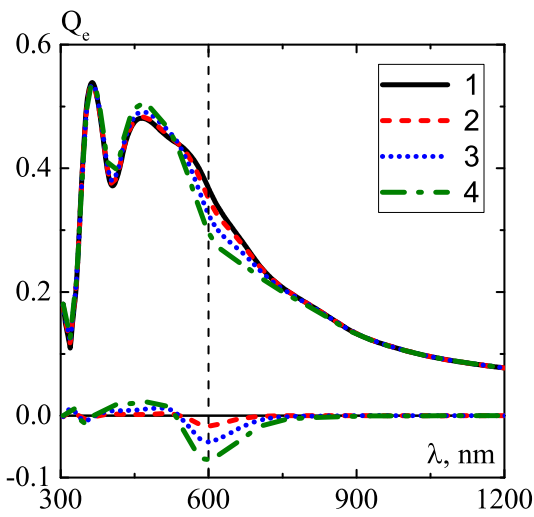

Fig. 11 Changes in the extinction spectrum of disordered nanoparticle aggregates composed of $50 \mathrm{Ag}$ nanoparticles with radiuses $10 \mathrm{~nm}$ (averaged over 300 aggregates): (1) the initial spectrum and the spectra after picosecond laser pulse $(\tau=20 \mathrm{ps}), \lambda=600 \mathrm{~nm}$ with the intensity: (2) $I=1.064 \cdot 10^{8} \mathrm{~W} \cdot \mathrm{cm}^{-2} ;(3)-I=1.663 \cdot 10^{8} \mathrm{~W} \cdot \mathrm{cm}^{-2}$; (4) $-I=2.395 \cdot 10^{8} \mathrm{~W} \cdot \mathrm{cm}^{-2} ;$ the corresponding differential spectra are shown at the bottom.

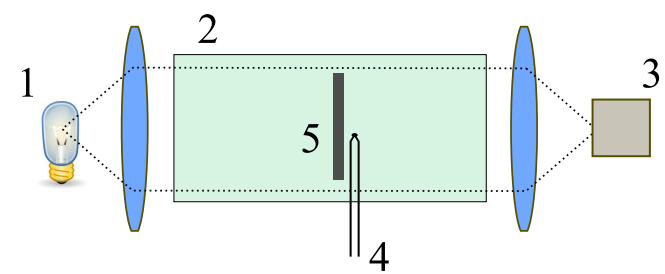

Fig. 121 - light source (250 W halogen lamp), 2 - quartz tube furnace, 3 - highspeed-spectrometer, 4 - K-type thermocouple, 5 - sample (quarz substrate with Au nanoparticles).

influence of radiation on the simplest particle configurations (dimer and trimer) allows one to predict the processes occurring in resonant domains of multiparticle aggregates. Fig. 11 shows dynamic changes in the extinction spectra of Ag multiparticle aggregates occurring under the action of picosecond laser pulses due to melting of a small fraction of particles in the domains. These changes are localized in resonant domains and manifest themselves as a spectral dip of limited spectral width $(\leq 100 \mathrm{~nm})$ in the plasmonic inhomogeneously broadened absorption spectrum which reproduces the experimental results $[42,43]$.

\section{Experimental}

To verify our calculations predicting suppression of the resonant properties of liquid plasmonic nanoparticles upon melting, we carried out an experiment with a sample containing $\mathrm{Au}$ nanoparticles resistant to oxidation in contrast to silver, copper and other plasmonic nanoparticles. Particles of $11 \mathrm{~nm}$ diameter were first deposited from monodisperse Au hydrosol onto a quartz substrate

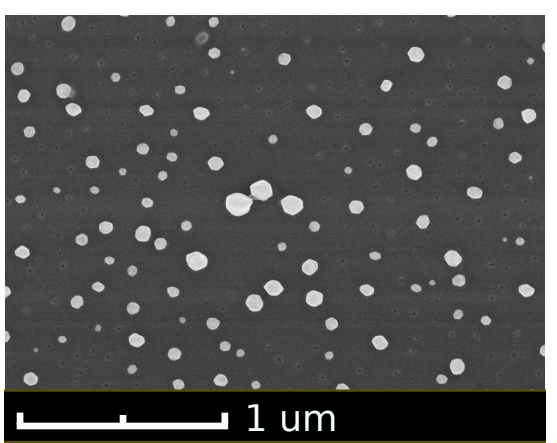

Fig. 13 SEM image of gold nanoparticles on a quartz substrate after heating in a furnace.

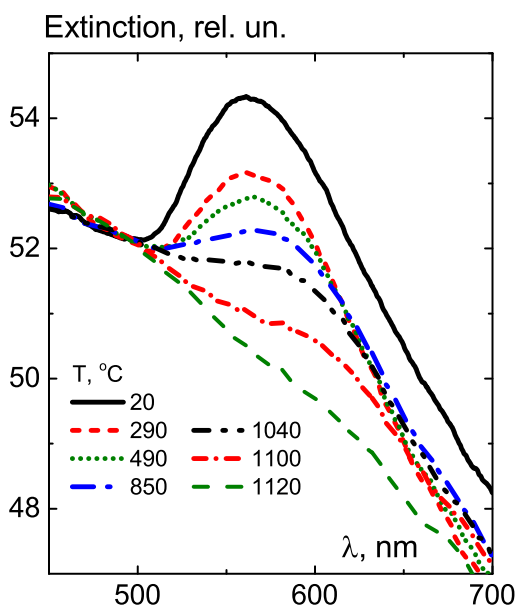

Fig. 14 Evolution of the absorption spectra of $\mathrm{Au}$ nanoparticles at room temperature (solid line) and at different temperatures up to $1120{ }^{\circ} \mathrm{C}$ (see the legend).

in arbitrary positions, with a small fraction of quaziordered small particle aggregates assembled during evaporation of water under the action of capillary and the van der Waals forces. The choice of Au colloids with this particular particle size was random and the initial size was not critical: the particles could be larger or smaller than this size to ensure the formation of particles of larger size (about $50 \mathrm{~nm}$ ) in the process of annealing. Fig. 12 shows the experimental setup with a tube furnace to heat the sample.

After the sample had been heated to $1100{ }^{\circ} \mathrm{C}$ and then annealed, the scanning electron microscopy (SEM) technique was applied to examine the sample structure, see Fig. 13. Our observations revealed that closely spaced initial gold nanoparticles in small aggregates were merged, forming larger polydisperse particles with an average diameter of $56 \mathrm{~nm}$ in random positions. These nanoparticles remain stable during several heating and annealing cycles. At the final stage the absorption spectrum of the sample was studied at different temperatures in the range $20-1120{ }^{\circ} \mathrm{C}$.

Fig. 14 shows the evolution of the absorption spectrum of $\mathrm{Au}$ nanoparticles on the substrate as the tem- 


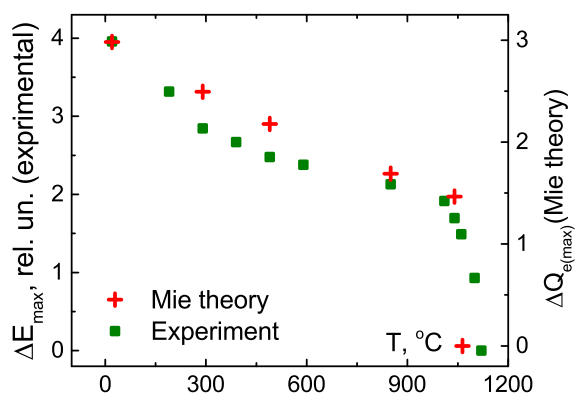

Fig. 15 Temperature dependences of the SPR peak amplitude of $\mathrm{Au}$ nanoparticles: comparison of experimental and computational results (obtained with approximation data for dielectric constant of gold from Section 2.2).

perature grows from room temperature and beyond the melting point up to $1120^{\circ} \mathrm{C}$.

One can see that the amplitude of the plasmon absorption peak gradually decreases with the growing temperature of the sample to result eventually in a complete suppression of SPR. The observed effect was reproduced in several heating cycles. The heating of nanoparticles is accompanied by a gradual shift of the maximum to longer wavelengths (within $50 \mathrm{~nm}$ ) and a simultaneous broadening of the absorption band. Fig. 15 shows the temperature dependence of the SPR amplitude in the extinction spectrum of the sample. At temperatures higher than $1010{ }^{\circ} \mathrm{C}$ there is significant and rapid downturn of this curve, which can be explained by an approach to the melting temperature for the nanoparticles $\left(\approx 1064{ }^{\circ} \mathrm{C}\right)$. The theoretical approximation was performed using conventional equations of the Mie theory (see [39]) with the dependence of scattered and inner fields on the radius of a particle and relative dielectric constants of its material obtained in Section 2.2. In a recent paper [25] Au nanoparticles were heated to temperatures below the melting point which resulted in the broadening of SPR and decrise of its amplitude. In [44] the same effect was observed at the melting point of $\mathrm{Au}$ nanoparticles with radii 2,8 and $15 \mathrm{~nm}$ when finite size effect plays an important role. Note that with these radii a significant contribution of this effect can obscure the contribution of heating. In our experiments we reached the temperature when SPR in Au nanoparticles was completely suppressed when contribution of the finite size effect was negligible.

\section{Conclusion}

To summarize the obtained results, we showed that strong heating of nanoparticles consisting of basic plasmonic materials ( $\mathrm{Ag}$ and $\mathrm{Au}$ ) is one of the key factors to be considered when building correct models in plasmonics. We would like to emphasize here that this factor has never been taken into account in optical calculations. Earlier we draw an attention to the fact that resonant properties of Ag nanoparticles at the SPR frequency abruptly deteriorate when their state of aggregation changes from solid to liquid. Moreover, an increase of the temperature beyond the melting point leads to a further reduction in the SPR amplitude, which indicates the development of additional processes that contribute to the growth of the relaxation constant. In paper [13] we showed how this effect can produce optical nonlinearity of nanoparticle aggregates and composite media containing them. Apart from obtaining experimental facts, we proposed an interpretation of the observed effects and compared them with theoretical calculations.

Obviously, the same pattern is expected to be observed during heating and melting of other plasmonic nanoparticles. We believe that the factor of nanoparticle melting under interaction with high-intensity optical radiation is critically important and covers a wide range of phenomena, including nonlinear optical processes occurring in interaction of nanoparticles with pulsed laser radiation.

This work was performed within the State contract of the RF Ministry of Education and Science for Siberian Federal University for scientific research in 2017-2019. The numerical calculations were performed using the MVS-1000 M cluster at the Institute of Computational Modeling, Siberian Branch, Russian Academy of Sciences.

\section{References}

1. U. Kreibig and M. Vollmer, Optical Properties of Metal Clusters (Springer-Verlag, Berlin, 1995).

2. Plasmonics and plasmonic metamaterials. Analysis and applications, edited by G. Shvets and I. Tsukerman (World Scientific, Singapore, 2011).

3. M. I. Stockman, Opt. Express 19, 22029 (2011).

4. V. V. Klimov, Nanoplasmonics (Fizmatlit, Moscow, 2009).

5. M. A. Garcia, Journal of Physics D: Applied Physics 44, 283001 (2011).

6. J. Reintjes, Nonlinear Optical Parametric Processes in Liquids and Gases (Elsevier Science, Oxford, 1984).

7. B. S. Luk'yanchuk, A. E. Miroshnichenko, M. I. Tribelsky, Y. S. Kivshar, and A. R. Khokhlov, New Journal of Physics 14, 093022 (2012).

8. G. Baffou, P. Berto, E. B. Ureña, R. Quidant, S. Monneret, J. Polleux, and H. Rigneault, ACS Nano 7, 6478 (2013), pMID: 23895209.

9. A. D. Phan, T.-L. Phan, and L. M. Woods, Journal of Applied Physics 114, 214306 (2013).

10. A. Alabastri, A. Toma, M. Malerba, F. D. Angelis, and R. P. Zaccaria, ACS Photonics 2, 115 (2015).

11. A. P. Gavrilyuk and S. V. Karpov, Appl. Phys. B 97, 163 (2009).

12. A. P. Gavrilyuk and S. V. Karpov, Appl. Phys. B 102, 65 (2010). 
13. A. E. Ershov, A. P. Gavrilyuk, S. V. Karpov, and P. N. Semina, Appl. Phys. B 115, 547 (2014).

14. C. Kittel, Introduction to Solid State Physics, 6th ed. (John Wiley \& Sons, Inc., New York, 1986).

15. L. D. Landau and L. P. Lifshitz, Electrodynamics of Continuous Media (Pergamon Press, Oxford, 1984).

16. M. V. Berry and I. C. Percival, Optica Acta 33, 577 (1986).

17. O. B. Wright, Phys. Rev. B 49, 9985 (1994).

18. C.-K. Sun, F. Vallée, L. H. Acioli, E. P. Ippen, and J. G. Fujimoto, Phys. Rev. B 50, 15337 (1994).

19. N. Del Fatti, C. Voisin, M. Achermann, S. Tzortzakis, D. Christofilos, and F. Vallée, Phys. Rev. B 61, 16956 (2000).

20. R. H. M. Groeneveld, R. Sprik, and A. Lagendijk, Phys. Rev. B 51, 11433 (1995).

21. T. Castro, R. Reifenberger, E. Choi, and R. P. Andres, Phys. Rev. B 42, 8548 (1990).

22. M. Otter, Zeitschrift für Physik 161, 539 (1961).

23. J. C. Miller, Philosophical Magazine 20, 1115 (1969).

24. K. Ujihara, Journal of Applied Physics 43, 2376 (1972).

25. O. Yeshchenko, I. Bondarchuk, V. Gurin, I. Dmitruk, and A. Kotko, Surface Science 608, 275 (2013).

26. O. A. Yeshchenko, I. M. Dmitruk, A. A. Alexeenko, A. V. Kotko, J. Verdal, and A. O. Pinchuk, Plasmonics 7, 685 (2012).

27. J. D’Ans, J. Bartels, P. T. Bruggencate, A. Eucken, G. Joos, W. A. Roth, H. Borchers, H. Hausen, K.-H. Hellwege, K. Schäfer, and E. Schmidt, Landolt-Börnstein: Zahlenwerte und Funktionen, 6 ed. (Springer-Verlag, Berlin, 1957), Vol. 4.

28. P. B. Johnson and R. W. Christy, Phys. Rev. B 6, 4370 (1972).

29. E. A. Taft and H. R. Philipp, Phys. Rev. 121, 1100 (1961).

30. G. S. Arnold, Appl. Opt. 23, 1434 (1984).

31. M. A. Ordal, L. L. Long, R. J. Bell, S. E. Bell, R. R. Bell, R. W. Alexander, and C. A. Ward, Appl. Opt. 22, 1099 (1983).

32. N. W. Ashcroft and N. D. Mermin, Solid State Physics (Saunders College, Philadelphia, 1976).

33. M. Bass and L. Liou, Journal of Applied Physics 56, 184 (1984).

34. S. R. Nagel and S. E. Schnatterly, Phys. Rev. B 9, 1299 (1974).

35. J. M. Ziman, Electrons and Phonons (Oxford University Press, Oxford, 1960).

36. C. Oubre and P. Nordlander, The Journal of Physical Chemistry B 108, 17740 (2004).

37. S. Underwood and P. Mulvaney, Langmuir 10, 3427 (1994).

38. S. Inasawa, M. Sugiyama, and Y. Yamaguchi, The Journal of Physical Chemistry B 109, 3104 (2005), pMID: 16851329 .

39. C. F. Bohren and D. R. Huffman, Absorption and Scattering of Light by Small Particles (John Wiley \& Sons, New York, 1998).

40. V. S. Gerasimov, A. E. Ershov, S. V. Karpov, A. P. Gavrilyuk, V. I. Zakomirnyi, I. L. Rasskazov, H. Ågren, and S. P. Polyutov, Optical Materials Express 7, 555 (2017).

41. V. A. Markel, V. M. Shalaev, E. B. Stechel, W. Kim, and R. L. Armstrong, Phys. Rev. B 53, 2425 (1996).
42. S. V. Karpov, A. K. Popov, and V. V. Slabko, Technical Physics 48, 749 (2003).

43. V. P. Safonov, V. M. Shalaev, V. A. Markel, Y. E. Danilova, N. N. Lepeshkin, W. Kim, S. G. Rautian, and R. L. Armstrong, Phys. Rev. Lett. 80, 1102 (1998).

44. D. Dalacu and L. Martinu, Appl. Phys. Lett. 77, 4283 (2000). 